\title{
Are attractive female voices really best characterized by feminine fundamental and formant frequencies?
}

\section{Stage 1 Registered Report Accepted in Principle}

\section{Evolution and Human Behavior}

Christoph Schild ${ }^{1}$, David R. Feinberg ${ }^{2}$, David A. Puts ${ }^{3}$, Julia Jünger ${ }^{4}$, Vanessa Fasolt ${ }^{5}$, Iris Holzleitner ${ }^{5}$, Kieran O'Shea ${ }^{5}$, Rebecca Lai ${ }^{5}$, Ruben Arslan ${ }^{6}$, Amanda Hahn ${ }^{7}$, Rodrigo A. Cárdenas $^{8}$, Lisa M. DeBruine ${ }^{5}, \&$ Benedict C. Jones ${ }^{5}$

\author{
${ }^{1}$ Department of Psychology \\ University of Copenhagen \\ Øster Farimagsgade 2A, 1353 Copenhagen, Denmark \\ ${ }^{2}$ Department of Psychology, Neuroscience, and Behaviour \\ McMaster University \\ Hamilton, Ontario L8S 4K1, Canada \\ ${ }^{3}$ Department of Anthropology \& Center for Brain, Behavior and Cognition \\ Pennsylvania State University \\ University Park, PA 16802, USA \\ ${ }^{4}$ Department of Psychology \& Leibniz Science Campus Primate Cognition \\ University of Goettingen \\ Gosslerstrasse 14, 37073 Goettingen, Germany \\ ${ }^{5}$ Institute of Neuroscience \& Psychology \\ University of Glasgow \\ Hillhead Street, Glasgow, Scotland, UK \\ ${ }^{6}$ Max Planck Institute for Human Development \\ Lentzeallee 94, 14195 Berlin, Germany \\ ${ }^{7}$ Department of Psychology \\ Humboldt State University \\ 1 Harpst Street, Arcata, CA 95519, USA \\ ${ }^{8}$ Department of Psychology \\ Pennsylvania State University \\ University Park, PA 16802, USA
}

Corresponding author: Christoph Schild (cs@ @sy.ku.dk) 


\begin{abstract}
Research into the characteristics of attractive women's voices has focused almost exclusively on associations with fundamental or formant frequencies. A recent study of a small sample of voices used a bottom-up approach to identify acoustic characteristics associated with women's vocal attractiveness, finding that many acoustic characteristics other than fundamental or formant frequencies predicted women's vocal attractiveness. Here we will replicate their methodology with a much larger sample of voices $(N=450)$ in order to identify vocal characteristics that reliably predict women's vocal attractiveness. Identifying such traits will provide new insights into and avenues for study of the possible function of vocal attractiveness.
\end{abstract}




\section{Are attractive female voices really best characterized by feminine fundamental and formant frequencies?}

\section{Introduction}

Vocal attractiveness is thought to influence important social outcomes, including mate choices (e.g. Apicella, Feinberg, \& Marlowe, 2007; Hill et al., 2013; Leongómez et al., 2014). Consequently, many studies have investigated the acoustic parameters that might characterize attractive voices. The majority of these studies have focused on the relationship between attractiveness and either fundamental frequencies (rate of vocal fold vibrations; Titze, 1994) or formant frequencies (supralaryngeal vocal tract resonances; Titze, 1994). These acoustic characteristics are sexually dimorphic (i.e., fundamental frequency and formant frequencies are typically higher in women's than men's voices, Puts et al., 2012, 2016). Consequently, many researchers have argued that lower fundamental frequency and formant frequencies in men and higher fundamental frequency and formant frequencies in women signal mate quality (Feinberg et al., 2005; Pisanski et al., 2015, 2016; Puts et al., 2012, 2016).

Correlational studies have reported that fundamental frequency is positively correlated with women's vocal attractiveness (e.g. Feinberg et al., 2008) and negatively correlated with men's vocal attractiveness (e.g. Šebesta et al., 2017). Some studies have also reported similar results for formant frequencies (Babel et al., 2014; Puts et al., 2016) and, at least in women, formant frequencies have generally predicted vocal attractiveness better than fundamental frequency (Babel et al., 2014; Puts et al., 2016). Studies in which fundamental frequency or formant frequencies were experimentally manipulated in voices have reported that increasing fundamental frequency or formant frequencies increased women's vocal attractiveness (Kandrik et al., 2016; Jones et al., 2010). By contrast, decreasing fundamental frequency or formant frequencies typically increased men's vocal attractiveness (Feinberg et al., 2005, 2011; Kandrik et al., 2016; Jones et al., 2010; Puts, 2005, 2006; Tsantani et al., 2016).

As outlined above, the majority of studies that investigated characteristics of attractive voices have focused on the potential role of fundamental or formant frequencies, since these are thought to signal mate quality. In other words, these studies used a top-down (i.e., theorydriven) approach that tested hypotheses about putative relationships between specific acoustic parameters on the assumption that these parameters signal mate quality. However, evidence for associations between indices of mate quality and both fundamental frequency and formant frequencies is rather mixed. For example, tests for correlations between these acoustic characteristics and potential markers of susceptibility to illness (e.g., immune response and factors thought to be associated with immune response) have reported both positive (Arnocky 
et al., 2018) and null (Skrinda et al., 2014) results. Moreover, results interpreted as evidence for associations between these vocal cues and health markers were among over fifty correlations and would not have been significant if alpha was corrected to reflect this high number of comparisons (Arnocky et al., 2018). Furthermore, studies on voice changes across the menstrual cycle find mixed and even contrary results (for an overview see Banai, 2017). These mixed results raise the possibility that changes in fundamental frequency, nor formant frequencies, are valid indicators of fertility across the cycle. In a similar vein, women's fundamental frequency and formant frequencies do not appear to be related to hormonal makers such as progesterone, estradiol, testosterone and cortisol (Puts et al., 2013; Puts et al., 2016). Importantly, links between these acoustic characteristics and other markers of phenotypic condition (body shape and/or size), although robust, are relatively weak (Pisanski et al., 2014, 2016). Less widely studied vocal parameters, such as harmonics-to-noise ratio and shimmer, also show robust relationships to female body morphology (Pisanski et al., 2016). Taken together, this mixed evidence suggests that systematic study of the roles of other vocal cues (e.g., harmonics-to-noise ratio and shimmer) will be important to move the field forward.

By contrast with the theory-driven approach described above, one study has used a more bottom-up (i.e., data-driven) approach in which a wider range of acoustic parameters was measured (e.g., harmonics-to-noise ratio, duration, spectral tilt, jitter, shimmer, cepstral peak prominence (CPP), energy). Next, these measures were subjected to principal component analyses to identify the dimensions of variance in the sample of voices (i.e., to identify principal components, PCs). Finally, the relationship between these PCs and attractiveness was tested (Babel et al., 2014). Babel et al. (2014) found that this PC-based model explained more variance in attractiveness than a top-down model that contained only fundamental frequency and formant frequencies (the latter estimated from standardized formant position). However, these results were based on only a very small sample of voices (30 male and 30 female voices). Indeed, Babel et al. (2014) described their study as exploratory. A similar approach was used to identify the characteristics of attractive voices by Collins and Missing (2003) and Collins (2000), but these studies also analyzed only small samples (30 female and 34 male voices, respectively). Such data driven approaches are recommended to establish whether results from theory-driven models are both robust and not outperformed by alternative accounts (Jack et al., 2018).

If Babel et al's (2014) results are robust, it would challenge current evolutionary models of vocal attractiveness by illustrating that fundamental frequency is a relatively poor 
predictor of vocal attractiveness and not necessarily the strong mate-quality signal it is often assumed to be. This would allow researchers to shift their focus away from studying the signal value of fundamental frequency and address the likely function of whatever acoustic characteristics emerge as being critical for attractiveness. Thus, establishing whether the Babel et al results are robust would be an important step toward developing new evolutionaryminded models of women's vocal attractiveness.

In light of the above, the current study will attempt to replicate Babel et al's (2014) results using a much larger sample of voices (450 adult women's voices). This is a much larger sample than in previous research on vocal attractiveness, giving us more power to detect weak relationships. We will focus on women's voices because, compared to men's voices, research on women's vocal attractiveness is arguably underrepresented in the published literature. Analyzing women's voices means our results will not speak to men's vocal attractiveness.

We will test four specific hypotheses.

Hypothesis 1. A simple linear regression model including mean fundamental frequency and standardized formant position as independent variables will significantly predict women's vocal attractiveness.

Hypothesis 2. The final model produced using a backwards selection regression model with a wider range of acoustic measures initially included as independent variables will significantly predict women's vocal attractiveness. Following Babel et al. (2014), the initial model will include the mean fundamental frequency, standard deviation of fundamental frequency, the PCs produced by a principal component analysis of formant frequencies, and the PCs produced by a principal component analysis of other acoustic measures (harmonicsto-noise ratio, duration, spectral tilt, jitter, shimmer, CPP, energy) as independent variables.

Hypothesis 3. The final model produced in our test of Hypothesis 2 will explain significantly more of the variance in vocal attractiveness than the model used to test Hypothesis 1.

Hypothesis 4. A model including mean fundamental frequency and the first PC produced by a principal component analysis of formant frequencies as independent variables will explain less variance in attractiveness than a model including the first two PCs produced by a principal component analysis of other acoustic measures (harmonics-to-noise ratio, duration, spectral tilt, jitter, shimmer, CPP, energy) as independent variables. 


\section{Methods}

\section{Stimuli}

Mono digital voice recordings of 450 young adult women (all students at the University of Glasgow) were taken using an Audio-Technica AT-4041 cardioid condenser microphone at a sampling rate of $44.1 \mathrm{kHz}$ at 16-bit amplitude quantization. Each participant was instructed to say "Hi, I'm a student at the University of Glasgow" in their normal speaking voice. These voice recordings have already been collected (as part of a large-scale study of hormonal regulation of women's mating psychology, Jones et al., 2018), but have not yet been analyzed or rated. The age range of women in our sample (university age students between 16 and 30 years of age) is similar to those of other studies investigating correlates of fundamental frequency and formant frequencies (e.g., Babel et al., 2014; Collins \& Missing, 2003; Feinberg et al., 2008; Pisanksi et al., 2014; Puts et al., 2012, 2016). Because there is little evidence that oral contraceptive alters speech qualities (for a review of 24 studies on this topic, see Rodney \& Sataloff, 2016), we do not plan to test for effects of oral contraceptive use. We will use the word "student" as stimuli because of research showing that attractiveness ratings of single words and sentences by the same speaker are highly similar (Mahrholz et al., 2018) and because it can reliably be parsed into its constituent vowel sounds for analysis of formants.

\section{Attractiveness ratings}

The sound pressure level of all voices will be amplitude normalized to $70 \mathrm{~dB}$ using the root mean squared method. Voice recordings will then be presented via headphones, all raters will rate each voice individually on attractiveness using a 1 (very unattractive) to 7 (very attractive) scale. Trial order will be fully randomized, participants will not be able to rate a voice until it has been played in full length. They will be able to listen to a voice as many times as they like before rating it (i.e., the rating task is self-paced). Participants will start each trial by clicking 'play'. Figure 1 shows a screen capture of the rating interface. Ninety participants (45 heterosexual men and 45 heterosexual women) will rate the voices. To avoid rater fatigue, each participant will be allocated to rate a set of 150 randomly selected voices only. Simulations have shown that $\sim 15$ raters per item is typically sufficient to obtain high agreement for attractiveness ratings (https://osf.io/x7fus/). 


\section{How attractive is this voice? \\ very unattractive (1) (2) (4) (4) (4) (4) attractive PLAY}

Figure 1. Interface for collecting vocal attractiveness ratings.

\section{Acoustic measures}

All acoustic measures will be taken following the procedures described in Babel et al. (2014). We will measure average fundamental frequency (f0) and standardized formant position for each speaker. Formant 1 (f1), formant 2 (f2), formant 3 (f3), and formant 4 (f4) will be measured from the recorded speech. Formants will be measured separately from both vowels in the word 'students' and averaged across vowels prior to further analysis. The standard deviation of $\mathrm{f} 0$ will also be calculated. f0 and formant frequencies will measured using Gaussian windows with a $2.5 \mathrm{~ms}$ step size. Formants will be measured at each glottal pulse and averaged across measurements. $\mathrm{f} 1, \mathrm{f} 2, \mathrm{f} 3$, and $\mathrm{f} 4$ will be used to calculate standardized formant position (following Puts et al., 2012, we will calculate this by standardizing each formant measure and dividing the sum of these four measures by four).

Harmonics-to-noise ratio (HNR) will be calculated in the $0-3.5 \mathrm{kHz}$ range for each voice. We will also measure duration (this will be measured from the onset to offset of spectral energy for each word and averaged for each talker), jitter (average of deviations of pitch periods), shimmer (average of deviations in amplitude of pitch periods), spectral tilt, CPP and energy. Spectral tilt is a measure of breathiness (Babel et al., 2014), while CPP indicates dysphonia severity (Fraile \& Godino-Llorente, 2014). Energy measures RMS (root mean squared) energy taken over a time series of pitch pulses (Shue, Keating, Vicenik, \& Yu, 2011). Spectral tilt will be measured using the same five measures used in Babel et al. (2014).

All acoustic measures besides Spectral tilt and CPP will be taken using Praat (Version 6.0.37; Boersma \& Weenink, 2018). The scripts for measuring F0, formants, jitter, shimmer and HNR are publicly available on the OSF (Feinberg, 2018, Puts \& Cardenas, 2018). Spectral til, CPP and energy will be measured using VoiceSauce (Shue, et al., 2011). 


\section{Data quality checks and exclusions}

Raters who give the same rating to $75 \%$ or more of the voices will be excluded from the dataset prior to any data analyses. Voices where the attractiveness rating or any acoustic measures is more than three standard deviations from the mean score for the sample on that measure will be excluded from the dataset prior to any data analyses. There will be no other exclusions. If the intraclass correlation coefficient (ICC 1,k; Shrout \& Fleiss, 1979) for attractiveness ratings is $<.8$, more raters will be added until the intraclass correlation coefficient for attractiveness ratings is $>.8$. Ratings by men and women will be combined (i.e., averaged) only if the correlation between men's and women's attractiveness ratings is greater than .80. Otherwise, men's and women's ratings will be analyzed separately, with more raters added until the intraclass correlation coefficient for attractiveness ratings is $>.8$.

Analyses

Analysis code for each of these tests is publicly available https://osf.io/8hma7.

Hypothesis 1. A simple linear regression model including mean fundamental frequency and standardized formant position as independent variables will significantly predict women's vocal attractiveness.

As in Babel et al. (2014), we will test this hypothesis using a simple linear regression analysis. The average attractiveness rating for each voice will be the dependent variable. Mean $\mathrm{f} 0$ and formant position will be the predictors. The largest sample of voices considered in a published correlational study of women's vocal attractiveness is 258 (Puts et al., 2016). Thus, our sample of 450 voices is considerably larger than the largest sample used previously to study women's vocal attractiveness.

Hypothesis 2. The final model produced using a backwards selection regression model with a wider range of acoustic measures initially included as independent variables will significantly predict women's vocal attractiveness. Following Babel et al. (2014), the initial model will include the mean f0, standard deviation of f0, the PCs produced by a principal component analysis of formant frequencies, and the PCs produced by a principal component analysis of other acoustic measures (harmonics-to-noise ratio, duration, spectral tilt, jitter, shimmer, CPP, energy) as independent variables.

As in Babel et al. (2014), we will first subject f1, f2, f3, and f4 measures to a PCA. We will then subject all other measures except mean f0 to a second PCA. We will select PCs for subsequent analyses using a parallel analysis to determine the number of factors to extract (Horn, 1965). As in Babel et al. (2014), we will use backwards selection regression to analyze these data. The average attractiveness rating for each voice will be the dependent variable. All 
selected PCs, mean f0, and standard deviation of $\mathrm{f0}$ will be predictors.

Hypothesis 3. The final model produced in our test of Hypothesis 2 will explain significantly more of the variance in vocal attractiveness than the model used to test Hypothesis 1.

Here we will use 10-fold cross validation with 100 repeats (i.e., 1000 resamples) to estimate the variance reliably explained by the model used to test Hypothesis 1 and the final model produced in our analyses for Hypothesis 2. We will then use an independent samples ttest to compare the proportion of variance explained by these two models. Sensitivity analysis using $\mathrm{G}^{*}$ Power (Faul, Erdfelder, Lang, \& Buchner, 2007) indicated that this test would have $95 \%$ power to detect an incremental variance explained of 0.02 at alpha $=.05$. Following Holzleitner et al. (2018), we will use the Akaike information criterion (AIC) to establish whether greater performance by the Hypothesis 2 model is simply due to overfitting.

Hypothesis 4. A model including mean fundamental frequency and the first PC produced by a principal component analysis of formant frequencies as independent variables will explain less variance in attractiveness than a model including the first two PCs produced by a principal component analysis of other acoustic measures (harmonics-to-noise ratio, duration, spectral tilt, jitter, shimmer, CPP, energy) as independent variables.

Here we will use 10-fold cross validation with 100 repeats (i.e., 1000 resamples) to estimate the variance reliably explained by the two models. We will then use an independent samples t-test to compare the proportion of variance explained by these two models. Sensitivity analysis using G*Power (Faul, Erdfelder, Lang, \& Buchner, 2007) indicated that this test would have $95 \%$ power to detect an incremental variance explained of 0.02 at alpha $=$ .05 .

\section{Additional analyses}

In addition to the analyses described above, we will also repeat each analysis including the quadratic terms for each predictor. We will then also test whether including these quadratic terms increases the predictive power of the models. For Hypotheses 3 and 4 we will also compare models using Akaike's information criterion (AIC) to establish whether the conclusions from the model comparisons in our main analyses generalize to another type of model comparison.

Some previous research found that fundamental frequency and formant frequencies interacted when women judged men's vocal attractiveness (Feinberg et al., 2011). Consequently, we will also test for an effect of the interaction between the linear effects of f0 and formant position on vocal attractiveness. 
If our main analyses are of combined ratings by male and female raters (see Data quality checks and exclusions, above, for criterion), we will repeat each analysis separately for male and female raters.

Analysis code for each of these additional analyses is publicly available at https://osf.io/8hma7/.

\section{References}

Apicella, C. L., Feinberg, D. R., \& Marlowe, F. W. (2007). Voice pitch predicts reproductive success in male hunter-gatherers. Biology Letters, 3, 682-684.

https://doi.org/10.1098/rsbl.2007.0410

Arnocky, S., Hodges-Simeon, C. R., Ouellette, D., \& Albert, G. (2018). Do men with more masculine voices have better immunocompetence? Evolution and Human Behavior, 39, 602610. https://doi.org/10.1016/j.evolhumbehav.2018.06.003

Boersma, P., \& Weenink, D. (2018). Praat: doing phonetics by computer [Computer program].Version 6.0.37, retrieved 11 October 2018 from http://www.praat.org/

Babel, M., McGuire, G., \& King, J. (2014). Towards a More Nuanced View of Vocal Attractiveness. PLOS ONE, 9, e88616. https://doi.org/10.1371/journal.pone.0088616

Banai, I. P. (2017). Voice in different phases of menstrual cycle among naturally cycling women and users of hormonal contraceptives. PLOS ONE, 12(8), e0183462. https://doi.org/10.1371/journal.pone.0183462 Borkowska, B., \& Pawlowski, B. (2011). Female voice frequency in the context of dominance and attractiveness perception. Animal Behaviour, 82, 55-59. https://doi.org/10.1016/j.anbehav.2011.03.024

Collins, S. A. (2000). Men's voices and women's choices. Animal Behaviour, 60, 773-780. https://doi.org/10.1006/anbe.2000.1523

Collins, S. A., \& Missing, C. (2003). Vocal and visual attractiveness are related in women. Animal Behaviour, 65, 997-1004. https://doi.org/10.1006/anbe.2003.2123

Faul, F., Erdfelder, E., Lang, A. G., \& Buchner, A. (2007). G* Power 3: A flexible statistical power analysis program for the social, behavioral, and biomedical sciences. Behavior research methods, 39, 175-191. https://doi.org/10.3758/BF03193146

Feinberg, D. R. (2018, November 6). Praat Scripts. https://doi.org/10.17605/OSF.IO/HUZ7D

Feinberg, D. R., Jones, B. C., DeBruine, L. M., O’Connor, J. J. M., Tigue, C. C., \& Borak, D. J. (2011). Integrating fundamental and formant frequencies in women's preferences for men's voices. Behavioral Ecology, 22, 1320-1325. https://doi.org/10.1093/beheco/arr134 
Feinberg, D. R., Jones, B. C., Little, A. C., Burt, D. M., \& Perrett, D. I. (2005). Manipulations of fundamental and formant frequencies influence the attractiveness of human male voices. Animal Behaviour, 69, 561-568. https://doi.org/10.1016/j.anbehav.2004.06.012

Fraile, R., \& Godino-Llorente, J. I. (2014). Cepstral peak prominence: A comprehensive analysis. Biomedical Signal Processing and Control, 14, 42-54.

https://doi.org/10.1016/j.bspc.2014.07.001

Hill, A. K., Hunt, J., Welling, L. L. M., Cárdenas, R. A., Rotella, M. A., Wheatley, J. R., ... Puts, D. A. (2013). Quantifying the strength and form of sexual selection on men's traits. Evolution and Human Behavior, 34, 334-341. https://doi.org/10.1016/j.evolhumbehav.2013.05.004

Holzleitner, I. J., Lee, A. J., Hahn, A., Kandrik, M., Bovet, J., Renoult, J. P., ... \& Jones, B. C. (2018). A data-driven model of women's facial attractiveness reliably outperforms theorydriven models. PsyArXiv.

Horn, J. L. (1965). A rationale and test for the number of factors in factor analysis. Psychometrika, 30, 179-185. https://doi.org/10.1007/BF02289447

Jack, R. E., Crivelli, C., \& Wheatley, T. (2018). Data-Driven Methods to Diversify Knowledge of Human Psychology. Trends in Cognitive Sciences, 22(1), 1-5.

https://doi.org/10.1016/j.tics.2017.10.002

Jones, B. C., Hahn, A. C., Fisher, C. I., Wang, H., Kandrik, M., Han, C., ... DeBruine, L. M. (2018). No Compelling Evidence that Preferences for Facial Masculinity Track Changes in Women's Hormonal Status. Psychological Science, 29, 996-1005.

\section{https://doi.org/10.1177/0956797618760197}

Jones, B. C., Feinberg, D. R., DeBruine, L. M., Little, A. C., \& Vukovic, J. (2010). A domainspecific opposite-sex bias in human preferences for manipulated voice pitch. Animal Behaviour, 79, 57-62. https://doi.org/10.1016/j.anbehav.2009.10.003

Kandrik, M., Hahn, A. C., Wincenciak, J., Fisher, C. I., Pisanski, K., Feinberg, D. R., ... Jones, B. C. (2016). Are Men's Perceptions of Sexually Dimorphic Vocal Characteristics Related to Their Testosterone Levels? PLOS ONE, 11(11), e0166855.

\section{https://doi.org/10.1371/journal.pone.0166855}

Leongómez, J. D., Binter, J., Kubicová, L., Stolařová, P., Klapilová, K., Havlíček, J., \& Roberts, S. C. (2014). Vocal modulation during courtship increases proceptivity even in naive listeners. Evolution and Human Behavior, 35, 489-496.

https://doi.org/10.1016/j.evolhumbehav.2014.06.008 
Mahrholz, G., Belin, P., \& McAleer, P. (2018). Judgements of a speaker's personality are correlated across differing content and stimulus type. PLOS ONE, 13, e0204991. https://doi.org/10.1371/journal.pone.0204991

Pisanski, K., Fraccaro, P. J., Tigue, C. C., O’Connor, J. J. M., Röder, S., Andrews, P. W., ... Feinberg, D. R. (2014). Vocal indicators of body size in men and women: a meta-analysis. Animal Behaviour, 95, 89-99. https://doi.org/10.1016/j.anbehav.2014.06.011

Pisanski, K., Jones, B. C., Fink, B., O’Connor, J. J. M., DeBruine, L. M., Röder, S., \& Feinberg, D. R. (2016). Voice parameters predict sex-specific body morphology in men and women. Animal Behaviour, 112, 13-22. https://doi.org/10.1016/j.anbehav.2015.11.008

Puts, D., \& Cardenas, R. (2018, December 3). Voice scripts. https://doi.org/10.17605/OSF.IO/K2BHS

Puts, D. A., Hill, A. K., Bailey, D. H., Walker, R. S., Rendall, D., Wheatley, J. R., ... RamosFernandez, G. (2016). Sexual selection on male vocal fundamental frequency in humans and other anthropoids. Proc. R. Soc. B, 283, 20152830. https://doi.org/10.1098/rspb.2015.2830

Puts, D. A., Bailey, D. H., Cárdenas, R. A., Burriss, R. P., Welling, L. L. M., Wheatley, J. R., \& Dawood, K. (2013). Women's attractiveness changes with estradiol and progesterone across the ovulatory cycle. Hormones and Behavior, 63, 13-19.

https://doi.org/10.1016/j.yhbeh.2012.11.007

Puts, D. A., Jones, B. C., \& DeBruine, L. M. (2012). Sexual Selection on Human Faces and Voices. The Journal of Sex Research, 49, 227-243.

https://doi.org/10.1080/00224499.2012.658924

Puts, D. A. (2005). Mating context and menstrual phase affect women's preferences for male voice pitch. Evolution and Human Behavior, 26, 388-397.

https://doi.org/10.1016/j.evolhumbehav.2005.03.001

Puts, D. A. (2006). Cyclic variation in women's preferences for masculine traits. Human Nature, 17, 114-127. https://doi.org/10.1007/s12110-006-1023-X

Rodney, J. P., \& Sataloff, R. T. (2016). The Effects of Hormonal Contraception on the Voice: History of Its Evolution in the Literature. Journal of Voice, 30(6), 726-730. https://doi.org/10.1016/j.jvoice.2015.08.014

Šebesta, P., Kleisner, K., Tureček, P., Kočnar, T., Akoko, R. M., Třebický, V., \& Havlíček, J. (2017). Voices of Africa: acoustic predictors of human male vocal attractiveness. Animal Behaviour, 127, 205-211. https://doi.org/10.1016/j.anbehav.2017.03.014

Shrout, P. E., \& Fleiss, J. L. (1979). Intraclass correlations: Uses in assessing rater reliability. Psychological Bulletin, 86(2), 420-428. https://doi.org/10.1037/0033-2909.86.2.420 
Shue, Y.-L., Keating, P.,Vicenik, C. ,\& Yu K. (2011) VoiceSauce: A program for voice analysis. Proceedings of the ICPhS XVII, 1846-1849.

Skrinda, I., Krama, T., Kecko, S., Moore, F. R., Kaasik, A., Meija, L., ... Krams, I. (2014). Body height, immunity, facial and vocal attractiveness in young men. Naturwissenschaften, 101, 1017-1025. https://doi.org/10.1007/s00114-014-1241-8

Titze, I. R. (1994). Principles of voice production. Englewood Cliffs, N.J: Prentice Hall.

Tsantani, M. S., Belin, P., Paterson, H. M., \& McAleer, P. (2016). Low Vocal Pitch Preference Drives First Impressions Irrespective of Context in Male Voices but Not in Female Voices. Perception, 45(8), 946-963. https://doi.org/10.1177/0301006616643675 